\title{
Facilitating Clinical Trials Participation of Low Socioeconomic Status Patients
}

\author{
Kyla N. Price ${ }^{a} \quad$ Alexis B. Lyons $^{\mathrm{b}} \quad$ Iltefat H. Hamzavi $^{\mathrm{b}} \quad$ Jennifer L. Hsiao ${ }^{c}$ \\ Vivian Y. Shi ${ }^{d}$ \\ ${ }^{a}$ College of Medicine, University of Illinois, Chicago, IL, USA; ${ }^{b}$ Department of Dermatology, Henry Ford Health \\ System, Detroit, MI, USA; ' $D$ Division of Dermatology, Department of Medicine, University of California, Los Angeles, \\ CA, USA; division of Dermatology, University of Arkansas for Medical Sciences, Little Rock, AR, USA
}

\section{Introduction}

Socioeconomic status represents social standing measured by the combination of education, income, and occupation [1]. Financial barriers and lack of education generate a number of obstacles to receiving adequate healthcare and treatment. A recent study exploring demographics of cancer clinical trial participants found that patients with an annual household income of less than USD 50,000 had 32\% lower odds of trial participation than patients with above USD 50,000 [2]. There is limited data reporting low socioeconomic status (LSES) and participation in dermatology clinical trials; however, it is not unlikely that financial barriers result in similar disparities. These findings support the need to represent and care for LSES groups in clinical trials.

When conducting clinical trials with patients of LSES, it should be acknowledged that they represent a potentially vulnerable group $[3,4]$, and thus should not be specifically targeted for participation, but rather be provided the same opportunity for clinical trial enrollment and an optimized participation experience. At the same time, it is also important to acknowledge the potential mutual benefits of clinical trial participation for LSES patients.
Clinical trial participation can provide an opportunity for access to investigative medication that may not otherwise be affordable or covered by insurance for patients with LSES. Clinical trials also provide an avenue for improved outcomes for patients with disease that is recalcitrant to standard treatments. Additionally, studies have shown improved health outcomes as a result of clinical trial participation [5]. The goal of this paper is to discuss current barriers for study participants with LSES and present potential strategies to help mitigate these gaps.

\section{Reduced Income}

While most clinical trials provide monetary compensation, LSES patients must determine whether the provided funds are an adequate alternative to work compensation. Higher compensation allows patients to allocate more time towards clinical trial participation and can help facilitate childcare or caring for elderly or disabled family members (Table 1). Additional compensation and budgeting also offsets hidden, out-of-pocket costs to study participation such as topical steroids, moisturizers, wound care supplies, and rescue medication costs needed during flares. karger@karger.com

(C) 2020 S. Karger AG, Basel

www.karger.com/drm

Karger!
Vivian Y. Shi

Hidradenitis Suppurativa Clinic

Department of Dermatology, University of Arkansas for Medical Sciences

4301 W. Markham St., \#576, Little Rock, AR 72205-7199 (USA)

vivian.shi.publications@gmail.com 
Table 1. Challenges and solutions for improving clinical trial enrollment for patients with low socioeconomic status

\begin{tabular}{ll}
\hline Challenges & Potential solutions \\
\hline Trial design & \\
Financial barriers & - Negotiate higher participant compensation with sponsors \\
& - Design study with minimal in-person visits \\
& - Account for miscellaneous costs to patients including transportation, childcare needs, missed work, \\
& emollients, etc. \\
& - Minimize lag between patient tasks and payout \\
& - Send participant payments regularly and promptly, rather than quarterly or at the end of study \\
& approval is often required) \\
& - Advertise on free public radio and TV programs in other major languages unique to a geographic re- \\
& gion (Spanish in the US; French in Canada, etc.) \\
& - Involve bilingual research staff \\
& - Ensure accessibility to interpreters
\end{tabular}

\section{Recruitment}

Low health literacy - Create informed consent forms, study brochures, and advertising materials at or below $6^{\text {th }}$ grade reading level

- Utilize the "teach back" method to ensure understanding

Inaccessibility/lack of awareness of trials
- Advertise trial opportunities in community events and facilities (schools, churches, recreation centers, primary care centers, free health clinics)

- Advertise to local physicians for direct patient referrals

- Increase awareness of study opportunities on free media: public radio, free TV stations, social media

Distrust of healthcare system

- Partner with physicians who practice at clinics in underserved communities or free clinics so information about available trials is provided by a more familiar and trusted source

- Recruit patients within partnership the physician has already established a trust and rapport

Limited trial participation from underserved/ LSES communities
- Trial patients share positive experiences, encourage other members in their community to participant

- Improve trust for future generations within an underserved community

- Galvanize community and patient support group leaders

\section{Trial conduct/compliance/retention}

Transportation and - Help arrange ride-share and public transportations

lodging challenges - Provide transit schedules

- Provide compensation for travel expenses: fuel and time

- Provide and arrange lodging for long distance travels

Inability to attend follow - Provide flexible follow-up schedule (before or after regular business hours, weekends, if needed) up visit due to schedule - Schedule several visits in advance to help patients plan ahead conflicts (with work, - Provide doctor's note to school or employer to excuse patient for attending appointment childcare, etc.)

\begin{tabular}{ll}
\hline Inability to complete & - Set frequent reminders, text, calls \\
e-diaries at home & - For participants without home internet access, provider free prepaid hotspot internet (include in budget \\
& negotiation with sponsor) \\
& - Lend participant a smart phone for diaries and trial-related communication (if not already provided by \\
& the sponsor) \\
& - Ensure e-diaries require a literacy level at or below the 6th grade
\end{tabular}

\section{After trial completion}

Loss of access to health- _ - Help participants get on governmental or supplemental insurance plan care provider

Loss of access to trial medication
- Assist participants to establish care with local free health clinics

- Facilitate enrollment in patient assistance programs (from non-profit or pharmaceutical companies) to pay for treatments that are comparable or replace trial medications

- Provide prescription and skin care product coupons 


\section{Lack of Insurance and Accessibility}

The US Census Bureau reports health insurance coverage based on income. In 2018, only $86.2 \%$ of people in households with less than USD 25,000 had insurance coverage, compared to $96.8 \%$ in households earning more than USD 150,000 [6]. Patients with lower income also have barriers to receiving specialty care, including dermatology [7]. With limited access to specialty care, these patients are not evaluated to receive diagnoses or considered for clinical trials. Additionally, some trial sites do not take uninsured or out-of-network insurance, such as Medicaid. Promoting inclusion of eligible patients despite their insurance status allows enrollment of more low-income, uninsured patients.

Since LSES patients are also less likely to receive consistent in-person care, promoting advertisement in more accessible medias including online platforms, social media, free TV networks, and radio stations can increase exposure. Advertising at other frequently utilized community facilities such as schools, churches, free health clinics, and recreation centers may also increase accessibility. Clinical trial teams should communicate and establish connections with community clinics and primary care centers that serve uninsured populations to facilitate direct referrals for eligible candidates.

Medical visits to establish care with the principal investigator can be costly especially for uninsured patients. If possible, uninsured potential trial candidates may be seen outside of clinic schedules by research coordinators and clinical trial staff to procure the medical records and history necessary to determine eligibility for enrollment. Eligible uninsured patients or patients outside of the health system can also be added to electronic medical records with a designation of "trial-status only" or with a note of "no billing to insurance."

It is also important to acknowledge the potential future complications following clinical trial participation for LSES patients given their lack of insurance and reduced income. While many trial protocols allow participants to continue to receive the study medication following completion of the comparative phase, after this extended time period ends, those with inadequate insurance may not have access to medication. Potential lack of future medication coverage is an important conversation to have with patients prior to clinical trial enrollment. Patient assistance programs from non-profit organizations or pharmaceutical companies and coupons may be potential solutions to help mitigate the loss of access to comparable medications.

Low Socioeconomic Status in Clinical Trials

\section{Transportation}

Transportation can also impose a substantial barrier to clinical trial participation. LSES patients experience obstacles to both affordable and efficient transportation to maintain their normal schedules and jobs. Increasing transportation budgets and reimbursement allows patients the opportunity to use faster and more efficient methods, such as ride-sharing services, to reach clinical trial sites. Also, by hosting clinical trial sites within areas that are accessible to different socioeconomic statuses, more financially diverse participants can have access.

Minimizing study visit frequency and consolidating to a more centralized location can further reduce participant traveling and expenses. Selecting one location that has all required equipment (e.g. X-ray, labs) or that minimizes the number of locations and visits can better accommodate patients with limited access to transportation.

\section{Lack of Job Flexibility}

Clinical trials often have very strict visit schedules. LSES patients do not always have jobs that allow for the flexibility to participate. Therefore, encouraging trial teams to have flexible visit schedules when possible including before or after work hours, during lunch, or on weekends can better accommodate patients with inflexible work schedules. Scheduling visits far in advance can also allow patients with less flexible schedules to request specific days and times off. Alternatively, providing school and work notes to trial participants can also help to mitigate potential negative consequences.

\section{Health Literacy}

Related to education, LSES shares a complex relationship with health literacy [8]. Lack of health literacy can result in apprehension towards clinical trial participation due to lack of familiarity with the benefits and risks. Educating patients on the goals, potential benefits, and specific risks of clinical trials can foster understanding and shared decision making. Additionally, promoting awareness of applicable clinical trial opportunities and discussing clinical trials as a potential backup option for when medications are not approved can also facilitate understanding and continuity of care. Sponsors and regulatory bodies can help promote patient understanding by providing trial information at or below a sixthgrade reading level to accommodate various educational 
backgrounds. Principal investigators and clinical trial staff can also facilitate transparency by discussing clinical trial information with unconvoluted language and utilizing the "teach back" method to ensure understanding [9].

Investments also have to be made in advance of trial participation. LSES populations had historically been studied in an extractive fashion (e.g., Tuskegee syphilis experiment). Trust can be fostered with support group meetings that include LSES participants to help build a sense of community. In addition, galvanizing community leaders to present the findings of the research back to their community in the presence of a medical professional can also build trust and transparency.

\section{Future Directions}

Little is known about the frequency of participation, outcomes, and experience of LSES patients in dermatology clinical trials. Future investigations are warranted to understand patient perspectives on clinical trial enrollment and to determine how to best accommodate LSES patients. Upon completion of clinical trials, participants should also be provided surveys to determine how their experience can be improved for future studies.

\section{Conclusion}

The recent surge in pipeline medications underscores the evolving therapeutic landscape at the forefront of dermatology. LSES patients with diseases recalcitrant to standard therapies can benefit from equal opportunity to participate in clinical trials. By implementing the methods provided, we can mitigate some of the barriers to clinical trial participation for LSES patients and contribute to improving outcomes and reducing health disparities.

\section{Key Message}

Strategies are needed to help mitigate barriers to clinical trial participation for patients of low socioeconomic status.

\section{Conflict of Interest Statement}

A.B.L. is a sub-investigator for Incyte, Beiersdorf, Unigen Inc., General Electric, Lenicura, Estee Lauder, Miragen, Biofrontera, Pfizer, and L'Oréal. I.H.H. is an investigator for Clinuvel, Galderma, Janssen Biotech, PCORI, Incyte, L'Oréal, Beiersdorf, Estee Lauder, Unigen Inc., Ferndale Healthcare Inc., Pfizer, Allergan, and Johnson \& Johnson, has served as a consultant for Pfizer, Johnson and Johnson, and Beiersdorf, is on AbbVie advisory board (noncompensated), is president of the HS Foundation (noncompensated), and is co-chair of the Global Vitiligo Foundation (noncompensated). V.Y.S. is a stock shareholder of Learn Health and has served as an advisory board member, investigator, and/or received research funding from Sanofi Genzyme, Regeneron, AbbVie, Eli Lilly, Novartis, SUN Pharma, LEO Pharma, Pfizer, Menlo Therapeutics, Burt's Bees, Altus Labs, GpSkin, the National Eczema Association, Global Parents for Eczema Research, the Foundation for Atopic Dermatitis, and Skin Actives Scientific. There were no incentives or transactions, financial or otherwise, relevant to this work. K.N.P. and J.L.H. have no conflicts of interest to declare relevant to this work.

\section{Funding Sources}

No funding was received for this study.

\section{Author Contributions}

All authors contributed to drafting and revision of the manuscript and approved the final version for submission.

\section{References}

1 Status S. American Psychological Association; Available from: https://www.apa.org/ topics/socioeconomic-status/

2 Unger JM, Gralow JR, Albain KS, Ramsey SD, Hershman DL. Patient Income Level and Cancer Clinical Trial Participation: A Prospective Survey Study. JAMA Oncol. 2016 Jan;2(1):137-9.

3 Denny CC, Grady C. Clinical research with economically disadvantaged populations. J Med Ethics. 2007 Jul;33(7):382-5.

4 Council NR. Medicine Io, Studies DoE, et al. The Arctic Aeromedical Laboratory's Thy- roid Function Study: A Radiological Risk and Ethical Analysis. Washington: National Academies Press; 2000.

5 Baker JR, Vandal AC, Yeoh J, Zeng I, Wong S, Ryan SN. Clinical trial participation improves outcome: a matched historical cohort study. Clin Trials. 2013 Oct;10(5):735-43.

6 Berchick ER, Upton RD. Health Insurance Coverage in the United States: 2018. Washington: US Census Bureau; November 2019; Available from: https:/census.gov/content/ $\mathrm{dam} /$ Census/library/publications/2019/ demo/p60-267.pdf
7 Ezeonwu MC. Specialty-care access for community health clinic patients: processes and barriers. J Multidiscip Healthc. 2018 Feb;11: 109-19.

8 Stormacq C, Van den Broucke S, Wosinski J. Does health literacy mediate the relationship between socioeconomic status and health disparities? Integrative review. Health Promot Int. 2019 Oct;34(5):e1-17.

9 Yen PH, Leasure AR. Use and Effectiveness of the Teach-Back Method in Patient Education and Health Outcomes. Fed Pract. 2019;36(6): 284-9. 